\title{
A mesenteric solid tumor with unusual features in a young male: A case report
}

\author{
YOSHITOSHI ICHIKAWA ${ }^{1}$, TAMAKI MAEDA ${ }^{2}$, GAKU MIZOJIRI ${ }^{1,3}$, \\ SATOSHI ISHIKAWA ${ }^{1}$, TAKAOMI HAGI ${ }^{1}$, KIMIAKI HATTORI ${ }^{2,3}$ and HIROSHI OKA ${ }^{1}$ \\ Departments of ${ }^{1}$ Surgery and ${ }^{2}$ Pathology, Moriguchi Keijinkai Hospital, Moriguchi, Osaka 570-0021; \\ ${ }^{3}$ Department of Pathology, Osaka Medical College, Takatsuki, Osaka 569-8686, Japan
}

Received November 18, 2016; Accepted July 4, 2017

DOI: $10.3892 /$ mco.2017.1344

\begin{abstract}
The current study presents a mesenteric mesenchymal tumor case, with unusual features in diagnostic imaging and histology. A 16-year-old male was admitted to the hospital with abdominal pain. Computed tomography (CT) revealed an abdominal mass, $2 \mathrm{~cm}$ in diameter. The results of contrast-enhanced CT, magnetic resonance imaging and ${ }^{18} \mathrm{~F}$-fluorodeoxyglucose positron emission tomography indicated no specific features suggestive of its histology. Two arteries branching from the superior mesenteric artery were observed feeding the hypervascular tumor. After endoscopic and other laboratory findings revealed no additional lesions, the lesion was diagnosed as a primary mesenteric tumor. As the possibility of malignancy and future bleeding from this tumor could not be ruled out, a resection of the tumor was performed. During the surgery, the tumor, which was well circumscribed and hypervascular, was located in the mesentery of the jejunum. The resected tumor did not exhibit typical histological characteristics, and was labeled as 'myxoid smooth muscle neoplasm of uncertain biologic potential'. At 2 years after surgery, the patient remained well without evidence of recurrence. As primary mesenteric tumors are rare, particularly in young patients, it is considered important that this type of unusual tumor be included in the differential diagnosis for mesenteric tumors.
\end{abstract}

\section{Introduction}

Primary tumors arising in the mesentery have been reported as rare tumors; however, small primary tumors are being identified

Correspondence to: Dr Tamaki Maeda, Department of Pathology, Moriguchi Keijinkai Hospital, 2-47-12 Yagumohigashi-cho, Moriguchi, Osaka 570-0021, Japan

E-mail: tamaki@nevins.jp

Abbreviations: CT, computed tomography; MRI, magnetic resonance imaging; GIST, gastrointestinal stromal tumors; SFT, solitary fibrous tumors; H-E, hematoxylin-eosin

Key words: mesenteric tumor, myxoid solid tumor, smooth muscle neoplasm more easily as diagnostic imaging systems have improved. These tumors are typically mesenchymal: Paraganglioma $(1,2)$, lipoma (3), Schwannoma (4,5), leiomyosarcoma (6), liposarcoma (7), osteosarcoma (8), gastrointestinal stromal tumors (GIST) (9), solitary fibrous tumors (SFT) (10), hemangioma (11), lymphangioma (12), smooth muscle tumors (10) and several types of cystic tumors (13). The majority of patients with primary tumors arising in the mesentery present with abdominal pain, and ultrasonography and/or abdominal computed tomography (CT) and/or magnetic resonance imaging (MRI) are performed. The most common symptoms of these mesenchymal tumors are abdominal pain, nausea, vomiting, abdominal distention and loss of appetite, depending on their location and size. In some cases, patients are asymptomatic and the tumors are identified during routine medical check-up. A definitive diagnosis may be confirmed using pathological examination. As these mesenchymal tumors are frequently difficult to be characterized as benign or malignant via imaging, it is important to accumulate evidence of cases in order to develop guidelines for surgery, particularly for small tumors. The present case reports a primary mesenteric solid tumor in a young patient, lacking typical features, not only on imaging but on pathological examination as well.

\section{Case report}

A 16-year-old Japanese male presented to the emergency room at Moriguchi Keijinkai Hospital (Osaka, Japan) with sudden onset of vomiting and severe lower-right abdominal pain. Following further vomiting, the pain subsided completely but later reappeared and was localized to the left of the navel. A CT scan of the abdomen detected an abnormal mass $2 \mathrm{~cm}$ in diameter with calcification at the left side of the aorta and the height of the lower end of the left kidney (Fig. 1A and $\mathrm{B}$ ). Contrast-enhanced $\mathrm{CT}$ revealed that the tumor was early enhanced in the central area, but not its marginal zone (Fig. 1C). MRI identified a hypointense signal similar to muscle tissue on T1-weighted images (Fig. 1D), and a hyperintensity within the lesion compared with the marginal zone on T2-weighted images (Fig. 1E). ${ }^{18} \mathrm{~F}$-fluorodeoxyglucose positron emission tomography revealed no abnormal uptake in the lesion (data not shown). Angiography image identified two arteries branching from the superior mesenteric artery feeding 
A

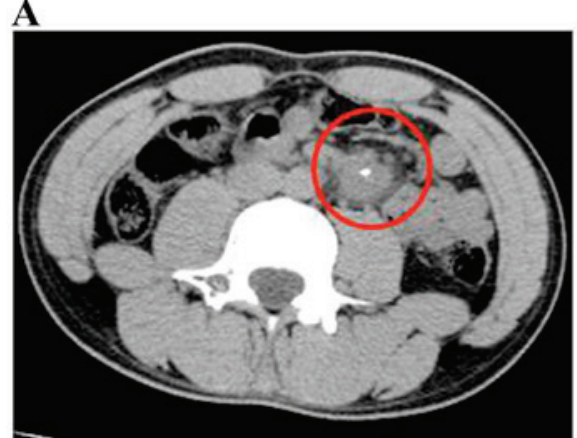

B
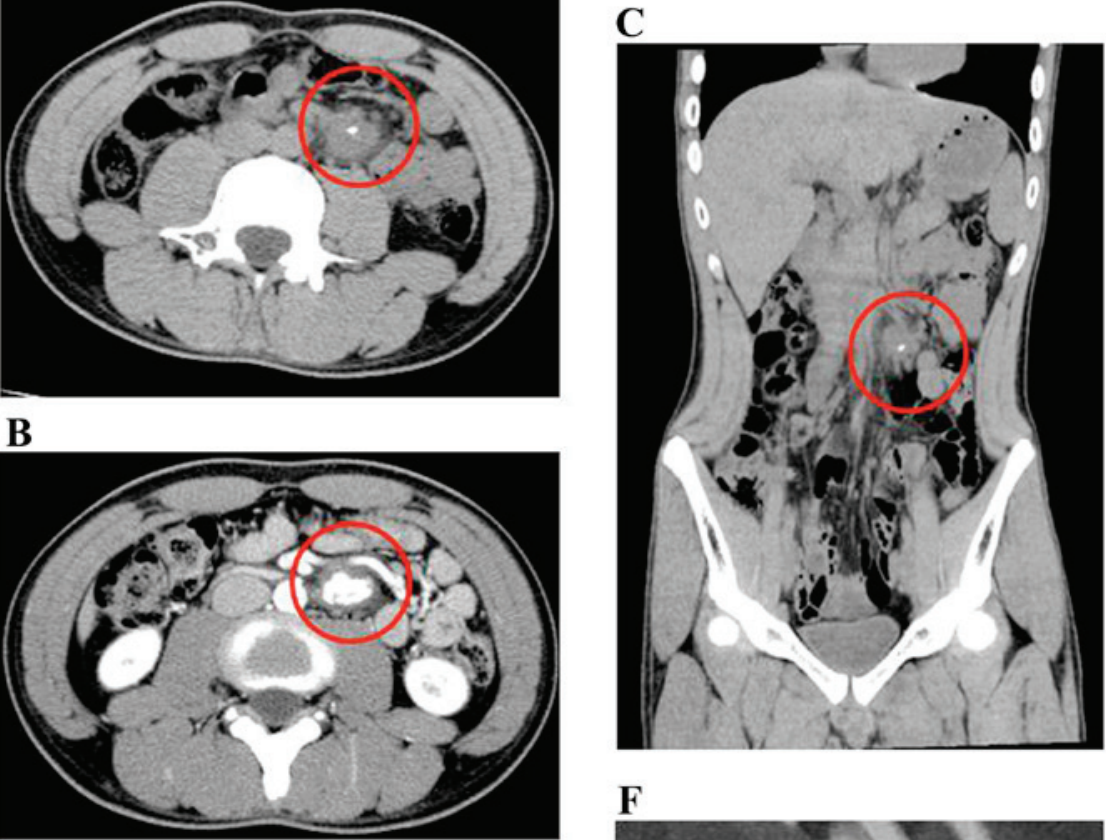

D

F

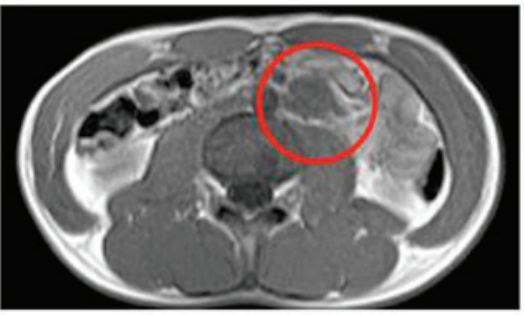

E
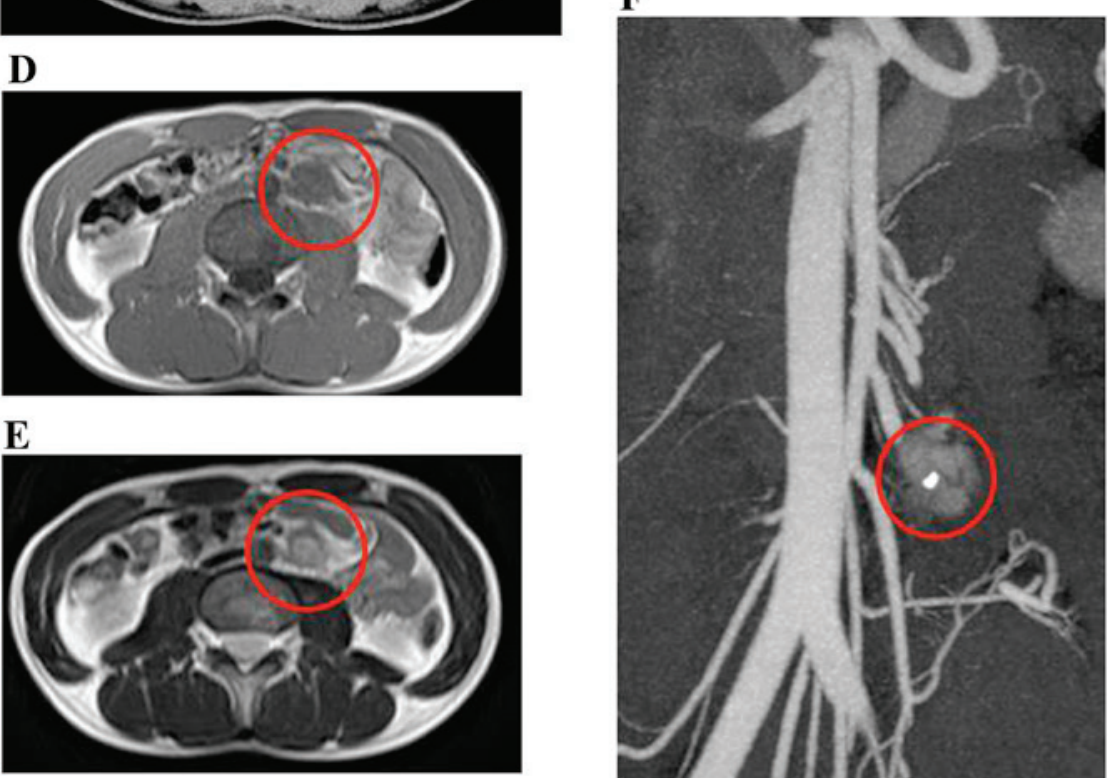

Figure 1. Radiological findings. (A) Axial and (B) coronal computed tomography (CT) images of the abdomen reveal an abnormal mass, 2 cm in diameter, with calcification. (C) Contrast-enhanced CT images showing early enhancement in the central area of the tumor, and a non-enhancement area in its marginal zone. Axial T2-weighted magnetic resonance images showing (D) hypointense signal on T1-weighted images and (E) a hyperintensity within the lesion compared with the marginal zone on the T2-weighted image. (F) On angiography, two tumor-feeding arteries branching from the superior mesenteric artery were identified.

the tumor (Fig. 1F). Tumor markers and other laboratory data suggested no specific diagnosis, and no lesions were identified by endoscopic examinations.

From these findings it was determined that the tumor originated from the mesentery. CT and contrast-enhanced CT scans suggested paraganglioma, Schwannoma, leiomyoma or leiomyosarcoma. There was, however, no tumor type that fulfilled the criteria of the MRI images for this case. As there was a risk of hemorrhage due to the hypervascularity of the lesion and as the possibility of malignancy was not able to be ruled out, laparoscopic excision of the tumor was performed following a preoperative suspected diagnosis of hemangioma.

During surgery, as the tumor was not located by endoscopy, an abdominal incision was made to investigate further.
A hypervascular tumor, $3 \mathrm{~cm}$ in diameter, was identified in the mesentery of jejunum at the anal side of the ligament of Treitz (Fig. 2A). The tumor had no capsule but was relatively circumscribed, and was easily removed along with surrounding adipose tissue. Certain lymph nodes around the tumor were also dissected. The frozen probe from the lymph nodes contained no atypical cells. The cut surface of the tumor was gray-white and yellow, surrounded by adipose tissue (Fig. 2B).

Histologically, the hematoxylin and eosin-stained tissue contained several components. The core of this lesion was a proliferation of spindle cells with cigar-shaped or tapering nuclei and strikingly elongated brightly eosinophilic cytoplasmic processes (Fig. 3A). These cells were distributed in a copious myxoid matrix, which contained scattered 
A

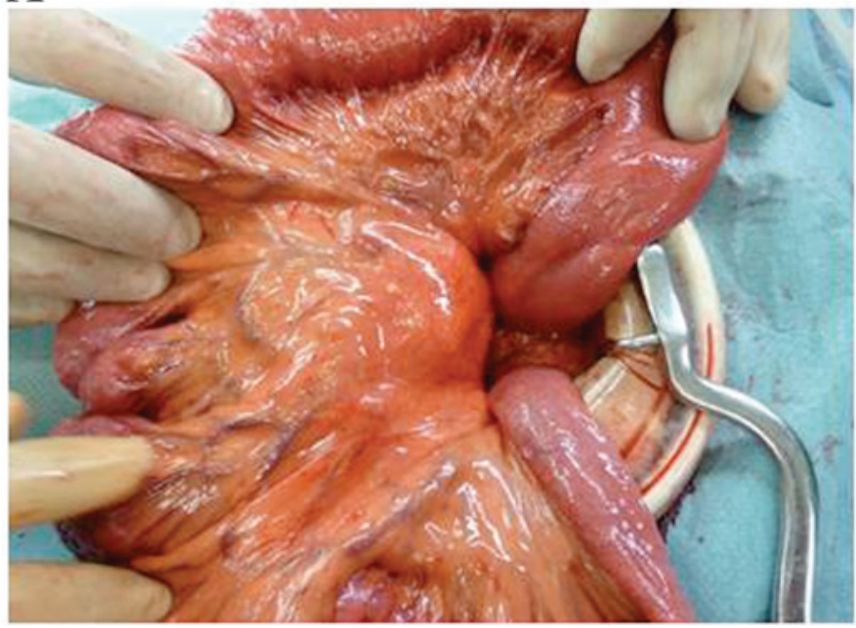

B

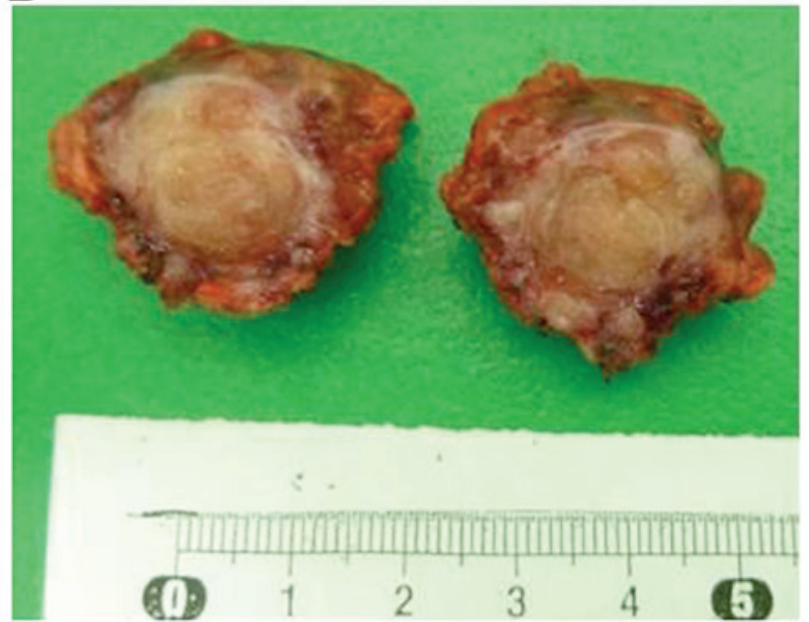

Figure 2. Intraoperative findings. (A) The mass was located in the mesentery of the jejunum. The 3-cm mass was totally excised. (B) Macroscopically, the mass was well-circumscribed.

A

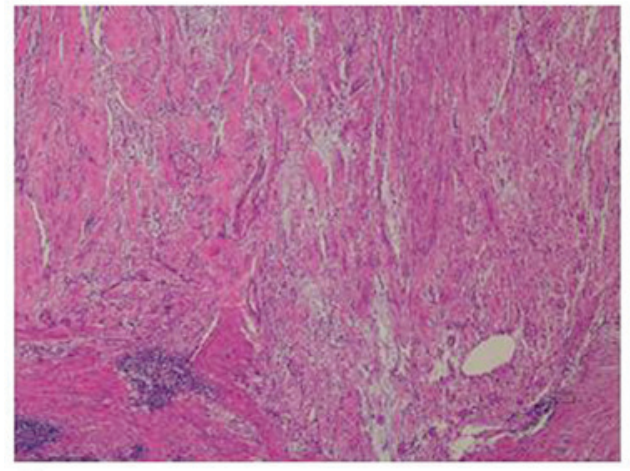

C

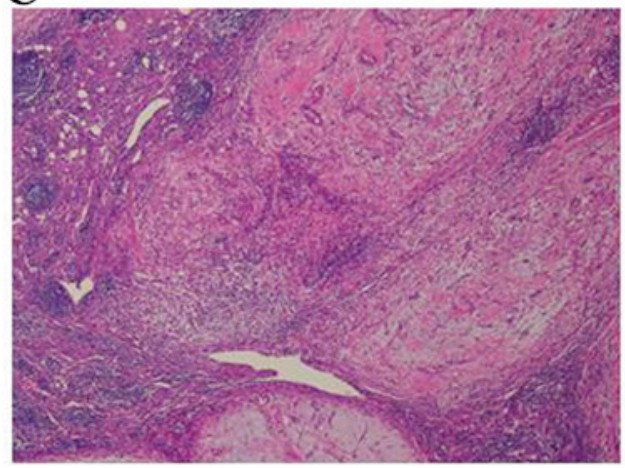

B

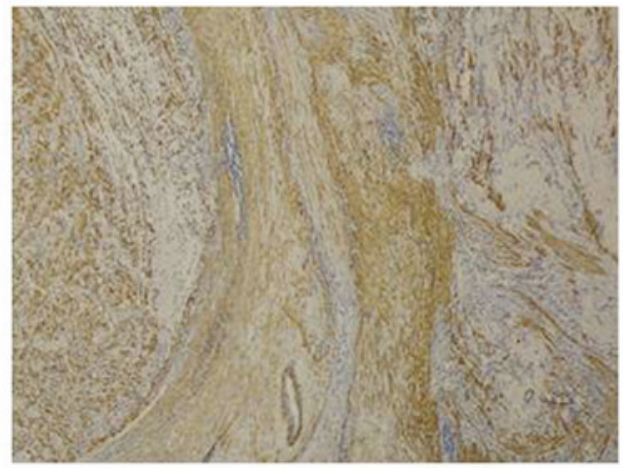

D

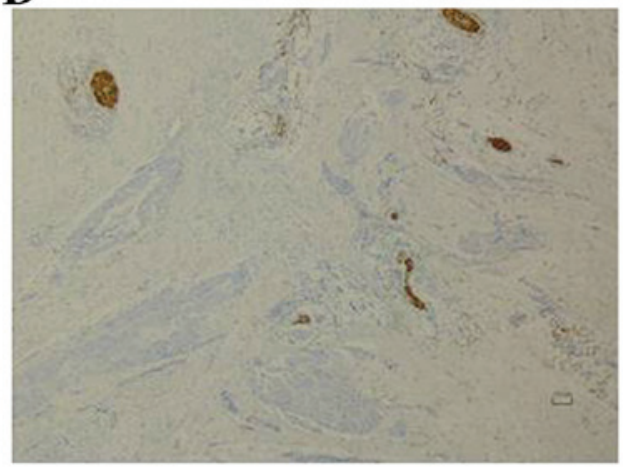

Figure 3. Histology and immunohistochemical analysis. (A) Hematoxylin and eosin staining revealed proliferation of spindle cells that were (B) positive for smooth muscle actin. (C) Numerous vessels and lymphoid tissues were observed around the tumor, as well as (D) nerves that were positive for S-100. Original magnification, $\mathrm{x} 200$.

lymphocytes. The calcification that was observed on CT imaging was not observed in the tissue preparation.

Immunohistochemical examination revealed that the spindle tumor cells were positive for smooth muscle actin (Fig. 3B), while negative for S-100 protein and anaplastic lymphoma kinase (data not shown). Numerous vessels and some nerve and lymphoid tissues were observed around the tumor (Fig. 3C and D). There was no significant atypia and mitotic figures were rare. From these histological findings, it was determined that the neoplastic cells were very likely to be of smooth muscle cell origin. However, a myxoid matrix with several vessels is not characteristic of leiomyoma or hemangioma. Following several consultations with pathologists experienced in soft tissue lesions, the tumor was labeled as 'myxoid smooth muscle neoplasm of uncertain biological potential'.

The patient has been well without any additional therapy, showing no evidence of recurrence for $>2$ years. Written informed consent was obtained from the patient for publication of this case report and any accompanying images. 


\section{Discussion}

Several types of mesenchymal tumors of mesenteric origin have been reported, including not only benign tumors, such as paraganglioma $(1,2)$, lipoma (3) and Schwannoma $(4,5)$, but also malignant lesions, such as leiomyosarcoma (6), liposarcoma (7) and osteosarcoma (8). In addition, there are certain mesenchymal tumors with high risk and poor prognosis, including GIST (9), solitary fibrous tumor (10), hemangioma (11), smooth muscle tumors (10), desmoid tumors (14) and fibromatosis (15). Neurofibromas in the mesentery have been reported, as in cases with von Recklinghausen's disease (16). Lymphomas and Castleman's disease must be also considered (17). In addition, other rare cases have been reported, including lymphangiomyomatosis (18), aggressive angiomyxoma (19) and non-neoplastic lesions, such as hamartoma (20), panniculitis (21), hematoma (22) and actinomycosis (23). As the ages of the patients vary, it is challenging to reach a diagnosis for such lesions according to the patient's age. Furthermore, as numerous tumors are difficult to diagnose preoperatively, pathological diagnosis of resected tumors is important for determining how to follow up the patient.

The previous case most similar to the present case was of a primary mesenteric smooth muscle tumor considered likely to behave in a malignant manner due to its large size of $20 \mathrm{~cm}$ in diameter (10). It is established that extrauterine smooth muscle tumors may exhibit a malignant behavior compared with uterine tumors, even if they do not exhibit aggressive histopathological characteristics. Although in our case the tumor was small, it was atypical; thus, a careful follow-up is required.

Alleviating symptoms is one of the goals of surgery, and in the current case the abdominal pain was relieved following surgery. The involvement of some nerve fibers in the proliferation of smooth muscle cells may have led to the wandering pain that was the chief complaint of the patient. The performed surgery was able to alleviate the pain experienced by the patient, in addition to preventing recurrence and hemorrhaging of the tumor.

In conclusion, the improvement of detection techniques is increasing the frequency of identification of small tumors in locations deeper within the body, such as the mesentery. Although the malignant potential in the present case remains unclear, we recommend that tumors with uncertain imaging diagnoses be resected and followed up closely, particularly in young patients, as there are numerous tumor types that are malignant or have malignant potential. It is necessary to accumulate pathological characteristics of cases with uncertain imaging diagnoses, until sufficient evidence is gathered to determine whether they are benign or malignant. The present case may contribute to considering the treatment of mesenteric tumors as one type of unclassified neoplasm.

\section{Acknowledgements}

The authors would like to thank Professor Christopher D.M. Fletcher, Department of Pathology, Harvard Medical School (Boston, USA), and Dr. Eiichi Konishi, Associate Professor, Department of Pathology, Kyoto Prefectural University of Medicine (Kyoto, Japan), for the diagnosis of the tumor.

\section{References}

1. Fujita T, Kamiya K, Takahashi Y, Miyazaki S, Iino I, Kikuchi H, Hiramatsu Y, Ohta M, Baba S and Konno H: Mesenteric paraganglioma: Report of a case. World J Gastrointest Surg 5: 62-67, 2013.

2. Ozkan Z, San Ozdemir C, Yasar G, Altas O, Koc M, Gul Y and Durdag E: An unusual mesenteric tumor 'paraganglioma': A case report. Iran Red Crescent Med J 16: e16837, 2014.

3. Ozel SK, Apak S, Ozercan IH and Kazez A: Giant mesenteric lipoma as a rare cause of ileus in a child: Report of a case. Surg Today 34: 470-472, 2004.

4. Kilicoglu B, Kismet K, Gollu A, Sabuncuoglu MZ, Akkus MA, Serin-Kilicoglu S and Ustun H: Case report: Mesenteric schwannoma. Adv Ther 23: 696-700, 2006.

5. Minami S, Okada K, Matsuo M, Hayashi T and Kanematsu T: Benign mesenteric schwannoma. J Gastrointest Surg 9: 1006-1008, 2005.

6. Sidhic AK, Ranjith M, Ali KP and Tej PR: Leiomyosarcoma of the mesentry, a rare mesentric tumour. Int J Surg Case Rep 7C: 58-60, 2015.

7. Sachidananda S, Krishnan A, Ramesh R and Kuppurao S: Primary multiple mesenteric liposarcoma of the transverse mesocolon. Ann Coloproctol 29: 123-135, 2013.

8. Heukamp LC, Knoblich A, Rausch E, Friedrichs N, Schildhaus HU, Kahl P, Tismer R, Schneider B, Büttner R and Houshdaran F: Extraosseous osteosarcoma arising from the small intestinal mesentery. Pathol Res Pract 203: 473-477, 2007.

9. Gorospe L, Simón MJ, Lima F, Esteban I, Madrid C and Hitos E: Primary mesenteric tumor with phenotypical features of gastrointestinal stromal tumors. Eur Radiol 12 (Suppl 3): S82-S85, 2002.

10. Kalogiannidis I, Stavrakis T, Amplianitis I, Grammenou S, Mavromatidis G and Rousso D: Primary mesenteric smooth muscle tumor: An entity with unpredictable biologic behavior. Case Rep Obstet Gynecol 2013: 483689, 2013.

11. Yang GZ, Li J and Jin H: Giant mesenteric hemangioma of cavernous and venous mixed type: A rare case report. BMC Surg 13: 50, 2013.

12. Chang SC, Tu SH, Shi MY, Huang SH and Chen KM: Mesenteric lymphangioma causing bowel obstruction: Report of one case. Acta Paediatr Taiwan 43: 43-45, 2002.

13. Liu Y, Peng Y, Li J, Zeng J, Sun G and Gao P: MSCT manifestations with pathologic correlation of abdominal gastrointestinal tract and mesenteric tumor and tumor-like lesions in children: A single center experience. Eur J Radiol 75: 293-300, 2010.

14. Ezumi K, Yamamoto H, Takemasa I, Nomura M, Ikeda M, Sekimoto M and Monden M: Dacarbazine-Doxorubicin therapy ameliorated an extremely aggressive mesenteric desmoid tumor associated with familial adenomatous polyposis: Report of a case. Jpn J Clin Oncol 38: 222-226, 2008.

15. McCormack D, Kesha K, Tittle SL and Saldinger PF: Mesenteric fibromatosis mimicking a gastrointestinal stromal tumor. Conn Med 74: 197-200, 2010.

16. Park J: Mesenteric plexiform neurofibroma in an 11-year-old boy with von Recklinghausen disease. J Pediatr Surg 42: E15-E18, 2007.

17. Papaziogas B, Chatzimavroudis G, Koutelidakis I, Grigoriou M and Atmatzidis K: A rare form of isolated mesenteric Castleman's disease presenting as an abdominal mass (isolated mesenteric Castleman's disease). J Gastrointestin Liver Dis 15: 171-174, 2006.

18. Mboyo A, Flurin V, Foulet-Roge A, Bah G, Orain I and Weil D: Conservative treatment of a mesenteric lymphangiomyomatosis in an 11-year-old girl with a long follow-up period. J Pediatr Surg 39: 1586-1589, 2004.

19. Kawachi J, Isogai N, Shimoyama R, Miyake K, Ogino H and Watanabe KA: Case of Mesentric Aggressive Angiomyxoma. J Jpn Surg Assoc 75: 1607-1610, 2014.

20. Nagae I, Hamasaki Y, Tsuchida A, Tanabe Y, Takahashi S, Minato S, Takada K, Koyanagi Y and Aoki T: Primary omental-mesenteric myxoid hamartoma of the mesoappendix incidentally detected after abdominal trauma in a child: Report of a case. Surg Today 35: 792-795, 2005.

21. Wen YK and Chen ML: Mesenteric panniculitis: An unusual cause of dyspepsia in a hemodialysis patient. Clin Nephrol 71: 224-227, 2009.

22. Vaiphei K, Gupta V and Rajesh LS: Mesenteric hematoma mimicking a neoplasm-a case report. Ann Diagn Pathol 10: 107-109, 2006.

23. Chan YL, Cheng CS and Ng PW: Mesenteric actinomycosis. Abdom Imaging 18: 286-287, 1993. 